\section{Dementia}

Accepted: September 5, 2010 Published online: January 20, 2011

\title{
Orbitofrontal Dysfunction Discriminates Behavioral Variant Frontotemporal Dementia from Alzheimer's Disease
}

\author{
M. Hornberger a, b S. Savage ${ }^{a} \quad$ S. Hsieh ${ }^{a} \quad$ E. Mioshi, ${ }^{a, b} \quad$ O. Piguet $^{a, b}$ \\ J.R. Hodges ${ }^{a, b}$
}

${ }^{a}$ Neuroscience Research Australia, and ${ }^{b}$ School of Medical Sciences, University of New South Wales, Sydney, N.S.W., Australia

\section{Key Words}

Orbitofrontal cortex · Disinhibition - Behavioral variant frontotemporal dementia • Alzheimer's disease

\begin{abstract}
Background: Behavioral variant frontotemporal dementia (bvFTD) patients show prefrontal cortex dysfunction and atrophy. Methods: We investigated whether executive function in conjunction with prefrontal cortex atrophy discriminates bvFTD and Alzheimer's disease (AD) patients efficiently at presentation. Results: AD and bvFTD patients were distinguishable by $89.5 \%$ on their performance of 3 executive tasks: the Hayling Test of Inhibitory Control, Digit Span Backward and Letter Fluency. Similarly, scan ratings showed that orbitofrontal cortex (OFC) and dorsolateral prefrontal cortex regions distinguish both patient groups. More importantly, employing the Hayling error score in conjunction with the OFC atrophy rating showed that $92 \%$ of patients can be correctly classified into bvFTD and AD. Conclusion: A combination of OFC and disinhibition measures appears to be a powerful diagnostic tool in differentiating bvFTD from $A D$ patients in this preliminary study.
\end{abstract}

Copyright ๑ 2011 S. Karger AG, Basel
(C) 2011 S. Karger AG, Basel

$1420-8008 / 10 / 0306-0547 \$ 26.00 / 0$

Fax +4161306 1234

E-Mail karger@karger.ch

www.karger.com
Accessible online at: www.karger.com/dem

\section{Introduction}

Clinical diagnostic criteria for the behavioral variant of frontotemporal dementia (bvFTD) have been proposed [1], but the differentiation from Alzheimer's disease (AD) remains difficult with the current neuropsychological and imaging tools [2]. On a neuropsychological level, discriminating bvFTD from $\mathrm{AD}$ on the basis of executive test performance has yielded inconsistent results $[3,4]$. However, recent findings show that bvFTD patients are impaired on a test of inhibitory function (Hayling) [5-7], which is a common behavioral feature seen in bvFTD at presentation [8]. This deficit has been reported to be orbitofrontal cortex (OFC)-dependent [9].

Moreover, neuroimaging studies have suggested that the brain regions most consistently involved in bvFTD are mesial/orbitofrontal prefrontal cortices [10], with OFC regions affected in the very early disease stages [11]. However, to date, OFC atrophy measures have been not considered in the clinical diagnosis of bvFTD.

This prospective study examined performance on the Hayling test and other executive function tests similar to [7] and their relation to a measure of brain atrophy using a previously validated MR visual rating scale [12]. More specifically, we investigated whether the Hayling test, which has been shown to recruit the OFC and adjacent 
Table 1. Mean scores (SD) for bvFTD patients, AD patients and controls on demographics, behavior and general cognitive tests

\begin{tabular}{|c|c|c|c|c|c|c|c|}
\hline $\mathrm{n}$ & 18 & 11 & 15 & - & - & - & - \\
\hline Age, years & $64.7(5.2)$ & $60.8(11.5)$ & $63.8(7.7)$ & n.s. & n.s. & n.s. & n.s. \\
\hline Education, years & $13.6(2.5)$ & $11.7(3.9)$ & $13.1(3.6)$ & n.s. & n.s. & n.s. & n.s. \\
\hline Sex, M/F & $9 / 9$ & $9 / 2$ & $11 / 4$ & - & - & - & - \\
\hline Length of history, years & - & $3.3(1.4)$ & $2.7(1.7)$ & - & - & - & n.s. \\
\hline Abnormal behavior & - & $11.3(7.7)$ & $4.1(4.3)$ & - & - & - & * \\
\hline Mood & - & $5.6(4.3)$ & $4.2(3.6)$ & - & - & - & n.s. \\
\hline Beliefs & - & $2.6(3.2)$ & $0.3(0.7)$ & - & - & - & * \\
\hline Stereotypic behavior & - & $9(6.2)$ & $4.8(4.6)$ & - & - & - & 0.06 \\
\hline Motivation & - & $8.4(7.3)$ & $7.1(6.1)$ & - & - & - & n.s. \\
\hline Total (180) & - & $135.5(78)$ & $95.8(63)$ & - & - & - & n.s. \\
\hline
\end{tabular}

F values indicate significant differences across groups; Tukey post-hoc tests compare differences between group pairs. n.s. $=$ Nonsignificant; ${ }^{*} \mathrm{p}<0.05{ }^{* *} \mathrm{p}<0.01 ;{ }^{* *} \mathrm{p}<0.001$.

prefrontal areas, could be used as an efficient discriminator between bvFTD and AD patients at presentation. In addition, we examined how orbitofrontal atrophy distinguishes the 2 patient groups. Finally, we considered the utility of a combination of executive tests and scan ratings in the discrimination of these groups.

\section{Methods}

\section{Case Selection}

Patients were selected from the FRONTIER Dementia Clinic database resulting in a sample of 11 bvFTD patients, $15 \mathrm{AD}$ patients and 18 controls. All bvFTD patients met current consensus criteria for FTD [1] and all AD patients met NINCDS-ADRDA diagnostic criteria [13] for probable $\mathrm{AD}$ (see table 1 for demographic details). All caregivers completed the Cambridge Behavioral Inventory [CBI, 14] to assess the behavioral symptoms. Ageand education-matched healthy controls were selected from a healthy volunteer panel, or were spouses/carers of patients.

Based on our prior findings [7], the following executive tests were administered: Brixton Spatial Anticipation test and Hayling tests, Digit Span, and the FAS Verbal Fluency and Trails test. A more detailed description of the tasks can be found in our previous study [7]. Task measures are listed in table 1.

Patients underwent general cognitive screening using the Addenbrooke's Cognitive Examination (ACE-R) [15] and MiniMental State Examination (MMSE). Only data from the first assessment were included in all analyses.

\section{Behavioral Analyses}

Data were analyzed using SPSS 17.0 (SPSS Inc., Chicago, Ill., USA). Parametric demographic (age, education), neuropsychological (executive and general cognitive tests) and behavioral $(\mathrm{CBI})$ data were compared across the 3 groups (bvFTD, AD and controls) via 1-way ANOVA followed by Tukey post-hoc tests. A priori variables were plotted and checked for normality of distribution by Kolmogorov-Smirnov tests. Variables revealing nonnormal distributions were log-transformed and the appropriate $\log$ values were used in the analyses. Variables showing nonparametric distribution after log transformation were analyzed via $\chi^{2}$, Kruskal-Wallis and Mann-Whitney U tests.

\section{Image Acquisition and Analysis}

All patient and controls underwent the same imaging protocol with a whole-brain T1-weighted images using a 3-tesla Philips MRI scanner with standard quadrature head coil (coronal orientation, matrix $256 \times 256,200$ slices, $1 \times 1 \mathrm{~mm}^{2}$ in-plane resolution, slice thickness $=1 \mathrm{~mm}, \mathrm{TE} / \mathrm{TR}=2.6 / 5.8 \mathrm{~ms}$, flip angle $\alpha=$ $\left.19^{\circ}\right)$

One rater (M.H.), who was blinded to the clinical diagnosis, rated T1 coronal MRIs based on a rating scale developed by Davies et al. [16] using a standard template against which to judge atrophy. The rater showed high reliability for the scoring of a MR training set of 100 scans (Cronbach's $\alpha=.95$ ). A more detailed description of the rating method can be found in the work of $\mathrm{Da}$ vies et al. [16]. Four prefrontal regions were scored: orbital, medial, dorsolateral and total prefrontal cortices. Atrophy within each region was rated on a 5-point Likert scale ranging from 0 to $4(0=$ normal, $4=$ severe atrophy; fig. 1$)$. The total prefrontal atrophy was obtained by averaging the atrophy ratings from the other 3 regions.
Hornberger/Savage/Hsieh/Mioshi/ Piguet/Hodges 
Fig. 1. Array of MR reference images and rating criteria employed in judging atrophy of the frontal lobe brain regions. Rating criteria range from $0=$ no atrophy, to 4 = severe atrophy for the 3 prefrontal brain regions $(\mathrm{OFC}=$ orbitofrontal cortex; $\mathrm{MPFC}=$ mesial prefrontal cortex; $\mathrm{DLPFC}=$ dorsolateral prefrontal cortex).

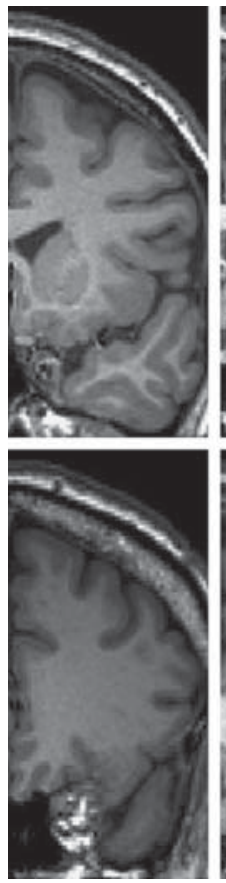

0

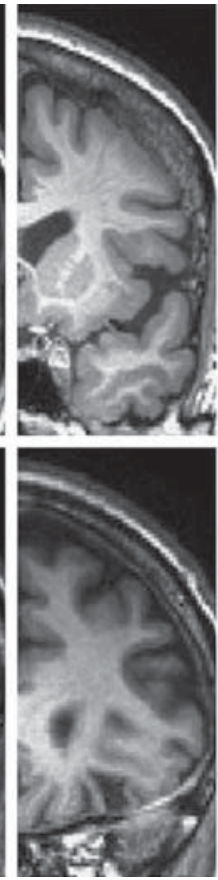

1
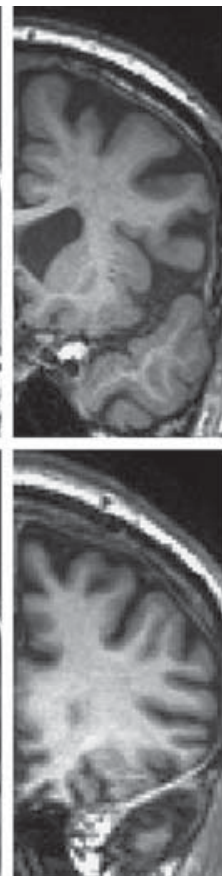

2

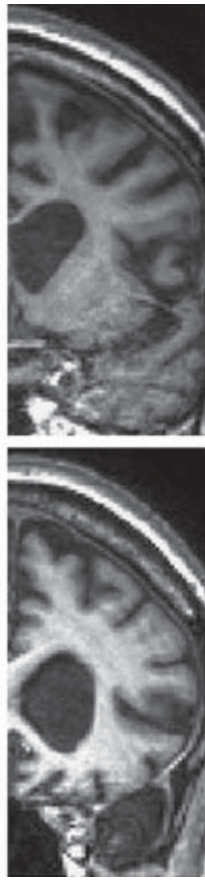

3
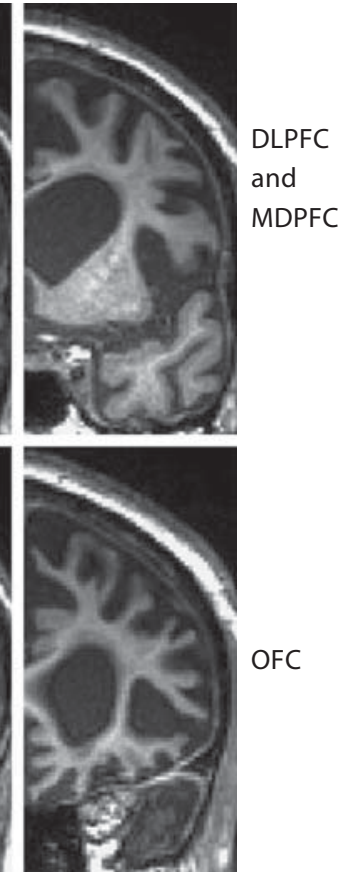

4

\section{Results}

Comparisons of bvFTD versus AD revealed no significant difference for any of the demographic variables. Controls were not significantly different from the 2 patient groups for any of the variables.

Performance of bvFTD and AD groups on the ACE-R and MMSE was generally worse than that of controls (table 1). Post-hoc tests showed that bvFTD and AD patients were equivalent for the MMSE score, whereas the bvFTD group was worse than AD on the ACE-R scores ( $\mathrm{p}<0.05)$. As evident from table 1, the bvFTD group showed higher levels of endorsement for the Abnormal Behavior, Beliefs and Stereotypic Behavior CBI subscores.

\section{Executive Function}

The results of the executive test are shown in table 2 . For Trails B and Brixton total scores, significant group main effects for time and error scores were present. Posthoc tests showed that bvFTD differed marginally from the controls on these measures, whereas AD patients were impaired in comparison to the controls. AD and bvFTD did not differ from each other (i.e. bvFTD $=\mathrm{AD}$ $<$ controls). By contrast, for the Hayling test, a significant main effect was present for both the overall scaled score and total error score. In addition, follow-up t tests re- vealed significant differences between bvFTD and controls and between $\mathrm{AD}$ and controls, as well as between bvFTD and AD on both scores (bvFTD $<\mathrm{AD}<$ controls).

For Digit Span, good differentiation between the bvFTD and AD groups was observed for the backward score only. On FAS Letter Fluency, bvFTD and AD groups were significantly different for the age scaled score, but not for the total correct responses.

In summary, performance on the tests of executive function was variable across tests. In other words, some tests did not discriminate bvFTD and AD groups well (Brixton and Trails), while others (Digit Span, Hayling and Letter Fluency) showed better diagnostic potential. A logistic regression analysis on the patient groups (bvFTD vs. AD) using the enter method was performed using task measures which showed a significant distinction between groups (Digit Span backward score, Hayling error score, Hayling overall score, FAS correct response and age scaled score). Overall, $89.5 \%$ of the patients could be correctly classified based upon these scores, with $79.2 \%$ distinguished by the Hayling error score alone (fig. 2a).

\section{MR Rating Analysis}

Analysis of the MRI ratings (table 3) showed that the groups differed on all atrophy measures. The bvFTD patients showed the most severe atrophy overall across all 

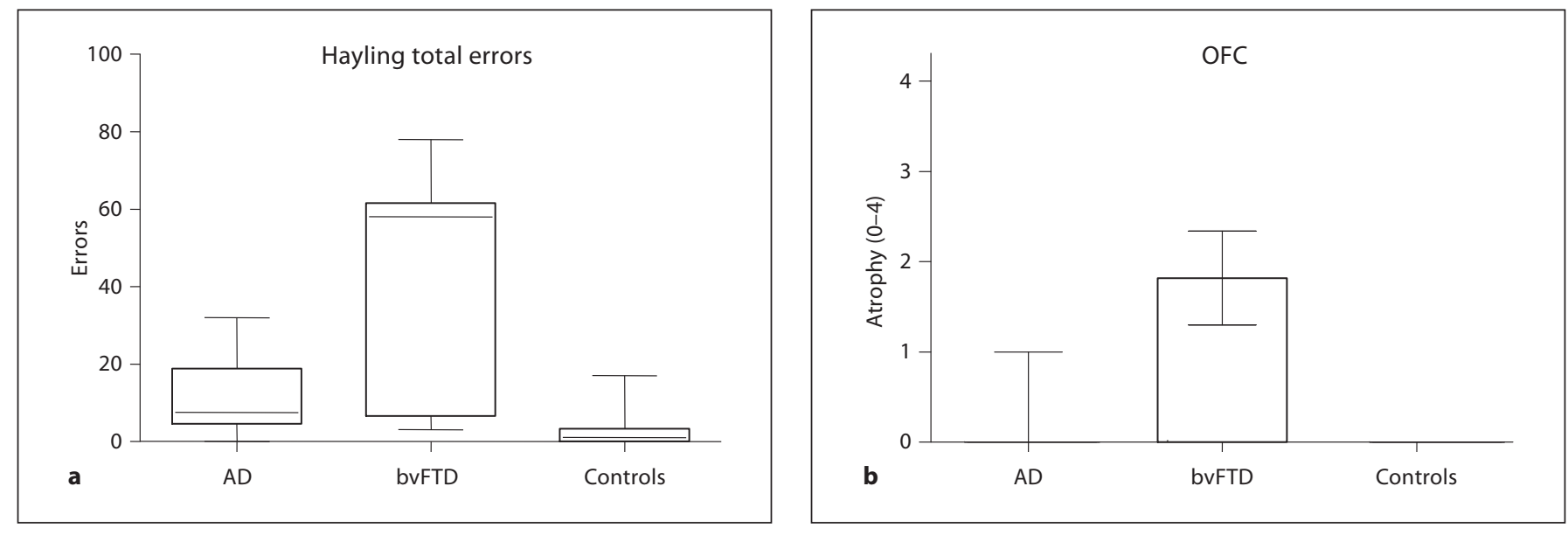

Fig. 2. Box plots of selected Hayling error scores as well as the atrophy score for the OFC brain regions across groups. Whiskers indicate the 5 th to 95 th percentiles.

Table 2. Mean scores (SD) for bvFTD, AD patients and controls on executive function tests

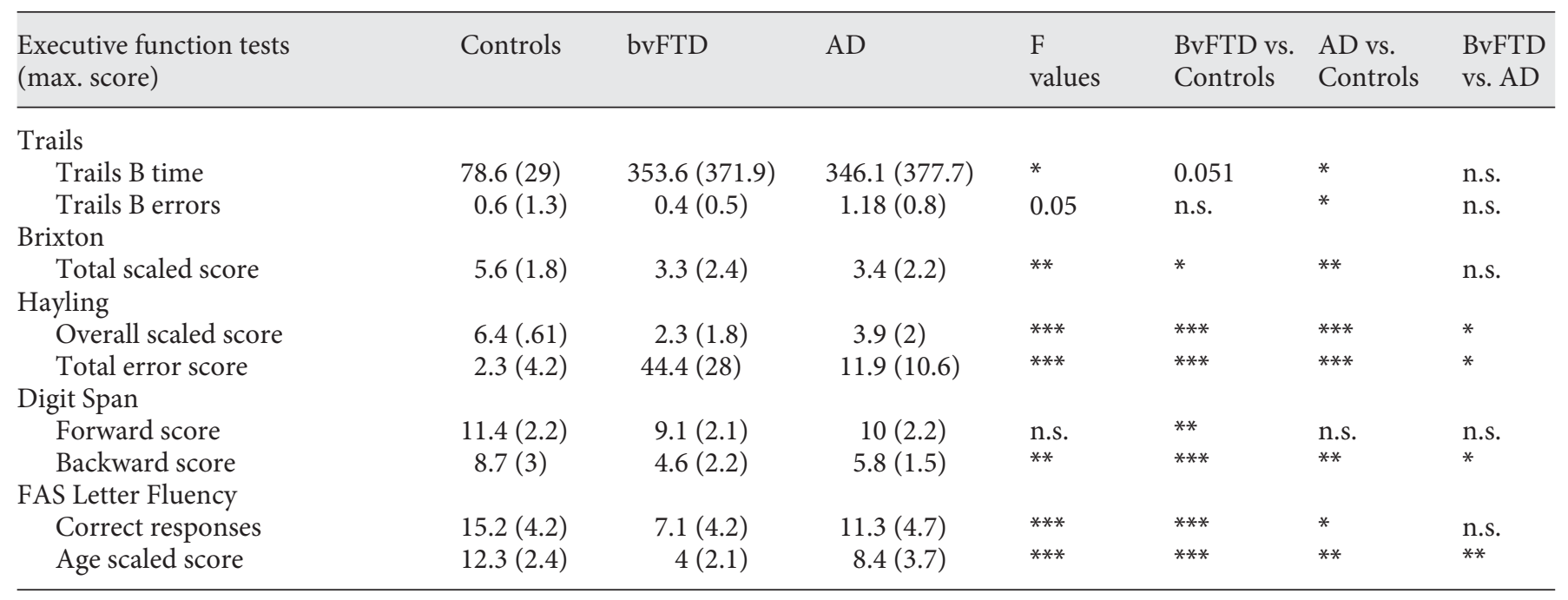

F values indicate significant differences across groups; Tukey post-hoc tests compare differences between group pairs. FAS age scaled score norms taken from Ivnik et al. [22]. n.s. = Nonsignificant; ${ }^{*} \mathrm{p}<0.05 ;{ }^{* *} \mathrm{p}<0.01 ;{ }^{* *} \mathrm{p}<0.000$.

Table 3. Mean scores (SD) for bvFTD patients, $\mathrm{AD}$ patients and controls on scan ratings

\begin{tabular}{|c|c|c|c|c|c|c|c|}
\hline Atrophy ratings (0-4) & Controls & bvFTD & $\mathrm{AD}$ & F values & $\begin{array}{l}\text { BvFTD vs. } \\
\text { Controls }\end{array}$ & $\begin{array}{l}\text { AD vs. } \\
\text { Controls }\end{array}$ & $\begin{array}{l}\text { BvFTD } \\
\text { vs. AD }\end{array}$ \\
\hline PFC & $0.56(0.46)$ & $2.3(1.1)$ & $1.2(0.71)$ & $* * *$ & $* * *$ & * & $* *$ \\
\hline OFC & 0 & $1.8(1.7)$ & $0.2(0.4)$ & $* * *$ & $* * *$ & n.s. & $* * *$ \\
\hline MPFC & $0.94(0.8)$ & $2.6(0.95)$ & $1.8(1)$ & $* * *$ & $* * *$ & $*$ & n.s. \\
\hline DLPFC & $0.72(0.67)$ & $2.5(1.1)$ & $1.6(1)$ & $* * *$ & $* * *$ & * & * \\
\hline
\end{tabular}

F values indicate significant differences across groups; Tukey post-hoc tests compare differences between group pairs. n.s. $=$ Nonsignificant; ${ }^{*} \mathrm{p}<0.05 ;{ }^{* *} \mathrm{p}<0.01 ;{ }^{* * *} \mathrm{p}<0.000$. 
measures. $\mathrm{AD}$ patients showed significant differences to controls for total prefrontal (PFC), mesial prefrontal (MPFC) and dorsolateral prefrontal (DLPFC) cortex regions, but not significantly for orbitofrontal regions. More importantly, bvFTD and AD groups differed for total PFC, DLPFC and OFC atrophy measures. In particular, the OFC ratings showed a very good discrimination of bvFTD and $\mathrm{AD}$, which was confirmed by a logistic regression analysis, showing that $73.1 \%$ of patients could be correctly classified on the OFC atrophy measure alone (fig. 2b).

\section{Sensitivity of Combined Measures for Group Membership}

In a final analysis, we conducted a logistic regression analysis with the 2 factors most sensitive to group membership (Hayling error score, OFC atrophy rating). We found that by employing the Enter method, $91.7 \%$ of cases were correctly classified into bvFTD and AD.

\section{Discussion}

Our study demonstrated that bvFTD and AD patients can be discriminated on the basis of their performance on specific executive function tests, with the Hayling Test of Inhibitory Function emerging as the most efficient discriminator. Visual MR scan ratings of PFC regions also distinguished patient groups. In particular, OFC atrophy is a good diagnostic indicator to discriminate bvFTD from AD patients and from controls. Together, the Hayling Error Score and OFC atrophy rating distinguished over $91 \%$ of cases correctly.

In keeping with prior results from a retrospective study of a separate cohort [7], the Hayling, Digit Span Backwards and Verbal Fluency test scores emerged as efficient indicators of group membership. This finding was further confirmed by the logistic regression analysis which showed that these scores correctly classified the majority of patients. In addition, a simple and quick visual MR rating scale [12] was capable of detecting PFC atrophy and discriminating bvFTD from AD patients. More importantly, the OFC region emerged as the most sensitive indicator of group membership: very few $\mathrm{AD}$ patients show atrophy of this brain region in the early stages of the disease, despite evidence of more generalized frontal atrophy $[17,18]$. The OFC region has been implicated in bvFTD on the basis of quantitative MRI measures [11], as well as by pathological studies [19]. Standardized neuropsychological tests which target the OFC region are rare. Therefore, the development of the Hay-

ling test [5], which taps into the dysfunction in this region, has important implications for the diagnosis of bvFTD patients.

Clinically, an accurate distinction between bvFTD and AD early in the course of the disease can be difficult, as many $\mathrm{AD}$ patients present with executive dysfunctions which make it difficult to distinguish them from bvFTD on that basis alone [20]. In addition, most bvFTD patients are impaired on tests of episodic memory to a similar magnitude in $\mathrm{AD}$, although these patients typically remain well orientated [21]. Our study emphasizes the importance of employing quantitative tests that tap into orbitofrontal dysfunction. Such tests, in combination with scan ratings of $\mathrm{PFC}$ regions, emerge as powerful tools to distinguish bvFTD and AD. Thus, abnormal scores on such orbitofrontal tests combined with atrophy in this region on MR strongly suggest FTD rather than $\mathrm{AD}$ pathology. It should be noted, however, that a small percentage of bvFTD patients (8.3\%) in our sample showed a normal Hayling performance and OFC scan rating.

It is important to note that even though the sensitivity of tests for detecting bvFTD was high, our results should be regarded as preliminary until they have been replicated in a bigger sample as well as in histopathologically confirmed cases. Furthermore, it will also be interesting to combine the Hayling test with nonverbal orbitofrontal-dependent tests to explore disinhibition in the language FTD subtypes, as well as contrasting them with the behavioral variant patients.

\section{Disclosure Statement}

This study was partly supported by a National Health and Medical Research Council (NHMRC) project grant (No. 510106). O.P. is supported by a NHMRC Clinical Career Development Award Fellowship (No. 510184). J.R.H. is supported by an Australian Research Council Federation Fellowship (FF0776229). S.H. is supported by an Australian Postgraduate Award.

References

Dement Geriatr Cogn Disord 2010;30:547-552
1 Neary D, Snowden JS, Gustafson L, Passant U, Stuss D, Black S, Freedman M, Kertesz A, Robert $\mathrm{PH}$, Albert M, Boone K, Miller BL, Cummings J, Benson DF: Frontotemporal lobar degeneration: a consensus on clinical diagnostic criteria. Neurology 1998;51: 1546-1554.
-2 Gregory CA, Orrell M, Sahakian B, Hodges JR: Can frontotemporal dementia and Alz- heimer's disease be differentiated using a brief battery of tests? Int J Geriatr Psychiatry 1997;12:375-383.


-3 Kramer JH, Jurik J, Sha SJ, Rankin KP, Rosen HJ, Johnson JK, Miller BL: Distinctive neuropsychological patterns in frontotemporal dementia, semantic dementia, and Alzheimer disease. Cogn Behav Neurol 2003;16: 211-218.

-4 Rahman S, Sahakian BJ, Hodges JR, Rogers RD, Robbins TW: Specific cognitive deficits in mild frontal variant frontotemporal dementia. Brain 1999;122:1469-1493.

5 Burgess P, Shallice T: The Hayling and Brixton Tests. Bury St. Edmunds, Thames Valley Test Company, 1997.

-6 Lough S, Kipps CM, Treise C, Watson P, Blair JR, Hodges JR: Social reasoning, emotion and empathy in frontotemporal dementia. Neuropsychologia 2006;44:950-958.

7 Hornberger M, Piguet O, Kipps C, Hodges JR: Executive function in progressive and nonprogressive behavioral variant frontotemporal dementia. Neurology 2008;71: 1481-1488.

8 Piguet O, Hornberger M, Shelley BP, Kipps CM, Hodges JR: Sensitivity of current criteria for the diagnosis of behavioral variant frontotemporal dementia. Neurology 2009; 72:732-737.

-9 Peters F, Perani D, Herholz K, Holthoff V, Beuthien-Baumann B, Sorbi S, Pupi A, Degueldre C, Lemaire C, Collette F, Salmon E: Orbitofrontal dysfunction related to both apathy and disinhibition in frontotemporal dementia. Dement Geriatr Cogn Disord 2006;21:373-379.
10 Rosen HJ, Gorno-Tempini ML, Goldman WP, Perry RJ, Schuff N, Weiner M, Feiwell R, Kramer JH, Miller BL: Patterns of brain atrophy in frontotemporal dementia and semantic dementia. Neurology 2002;58:198208.

11 Seeley WW, Crawford R, Rascovsky K, Kramer JH, Weiner M, Miller BL, GornoTempini ML: Frontal paralimbic network atrophy in very mild behavioral variant frontotemporal dementia. Arch Neurol 2008;65: 249-255.

12 Kipps CM, Davies RR, Mitchell J, Kril JJ, Halliday GM, Hodges JR: Clinical significance of lobar atrophy in frontotemporal dementia: application of an MRI visual rating scale. Dement Geriatr Cogn Disord 2007;23: 334-342.

13 McKhann G, Drachman D, Folstein M, Katzman R, Price D, Stadlan EM: Clinical diagnosis of Alzheimer's disease: report of the NINCDS-ADRDA Work Group under the auspices of Department of Health and $\mathrm{Hu}$ man Services Task Force on Alzheimer's Disease. Neurology 1984;34:939-944.

14 Wedderburn C, Wear H, Brown J, Mason SJ, Barker RA, Hodges J, Williams-Gray C: The utility of the Cambridge Behavioural Inventory in neurodegenerative disease. J Neurol Neurosurg Psychiatry 2008;79: 500-503.

15 Mioshi E, Dawson K, Mitchell J, Arnold R, Hodges JR: The Addenbrooke's Cognitive Examination Revised (ACE-R): A brief cognitive test battery for dementia screening. Int J Geriatr Psychiatry 2006;21:1078-1085.
6 Davies RR, Kipps CM, Mitchell J, Kril JJ, Halliday GM, Hodges JR: Progression in frontotemporal dementia: identifying a benign behavioral variant by magnetic resonance imaging. Arch Neurol 2006;63:16271631.

17 Whitwell JL, Jack CR Jr: Comparisons between Alzheimer disease, frontotemporal lobar degeneration, and normal aging with brain mapping. Top Magn Reson Imaging 2005; 16:409-425.

18 Whitwell JL, Shiung MM, Przybelski SA, Weigand SD, Knopman DS, Boeve BF, Petersen RC, Jack CR Jr: MRI patterns of atrophy associated with progression to $\mathrm{AD}$ in amnestic mild cognitive impairment. Neurology 2008;70:512-520.

19 Kril JJ, Macdonald V, Patel S, Png F, Halliday GM: Distribution of brain atrophy in behavioral variant frontotemporal dementia. J Neurol Sci 2005;232:83-90.

-20 Kramer JH, Jurik J, Sha SJ, Rankin KP, Rosen HJ, Johnson JK, Miller BL: Distinctive neuropsychological patterns in frontotemporal dementia, semantic dementia, and Alzheimer disease. Cogn Behav Neurol 2003;16:211-218.

21 Hornberger M, Piguet O, Graham A, Nestor PJ, Hodges JR: How preserved is episodic memory in behavioral variant frontotemporal dementia? Neurology 2010;74:472-479.

-22 Ivnik RJ, Malec JF, Smith GE, Tangalos EG, Petersen RC: Neuropsychological tests' norms above age 55: COWAT, BNT, MAE token, WRAT-R reading, AMNART, STROOP, TMT, and JLO. Clin Neuropsychol 1996;10:262-278. 